\title{
Corrigendum to "Start-Up Characteristics of a Granule-Based Anammox UASB Reactor Seeded with Anaerobic Granular Sludge”
}

\author{
Lei Xiong, ${ }^{1,2}$ Yun-Yan Wang, ${ }^{1,2}$ Chong-Jian Tang, ${ }^{1,2}$ Li-Yuan Chai, ${ }^{1,2}$ Kang-Que Xu, ${ }^{1,2}$ \\ Yu-Xia Song, ${ }^{1,2}$ Mohammad Ali, ${ }^{1,2}$ and Ping Zheng ${ }^{3}$ \\ ${ }^{1}$ School of Metallurgy and Environment, Central South University, Changsha 410083, China \\ ${ }^{2}$ National Engineering Research Center for Control and Treatment of Heavy Metal Pollution, Changsha 410083, China \\ ${ }^{3}$ Department of Environmental Engineering, Zhejiang University, Zijingang Campus, Hangzhou 310058, China
}

Correspondence should be addressed to Chong-Jian Tang; chjtang@csu.edu.cn

Received 19 October 2017; Accepted 29 October 2017; Published 7 November 2017

Copyright (C) 2017 Lei Xiong et al. This is an open access article distributed under the Creative Commons Attribution License, which permits unrestricted use, distribution, and reproduction in any medium, provided the original work is properly cited.

In the article titled "Start-Up Characteristics of a GranuleBased Anammox UASB Reactor Seeded with Anaerobic Granular Sludge" [1], there were two errors in Figure 2, where
$\mathrm{NH}_{3}{ }^{+}$in the legend of Figure 2(a) should be changed to $\mathrm{NH}_{4}{ }^{+}$ and the $x$-axis in Figure 2(e) should be changed from " $\mathrm{pH}$ " to "Ratio." The corrected figure is as follows.

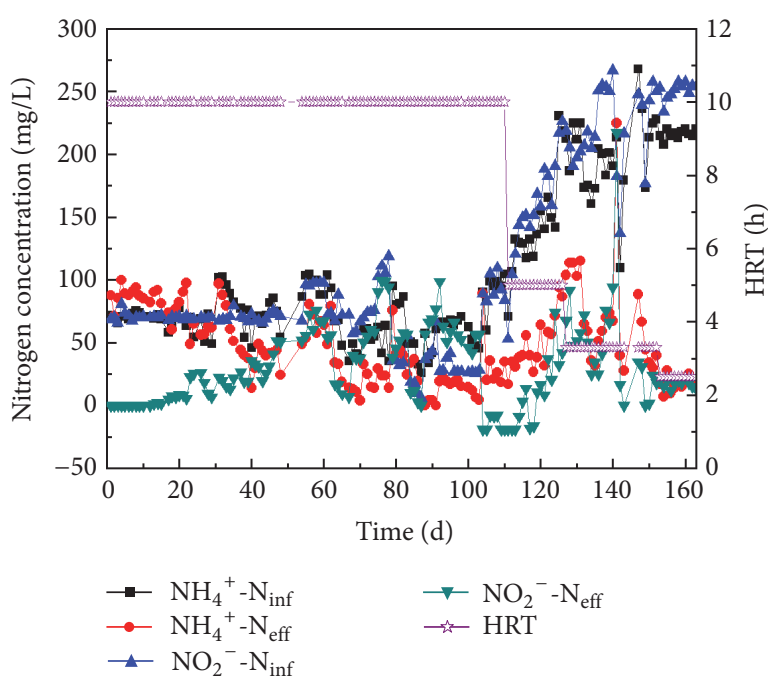

(a)

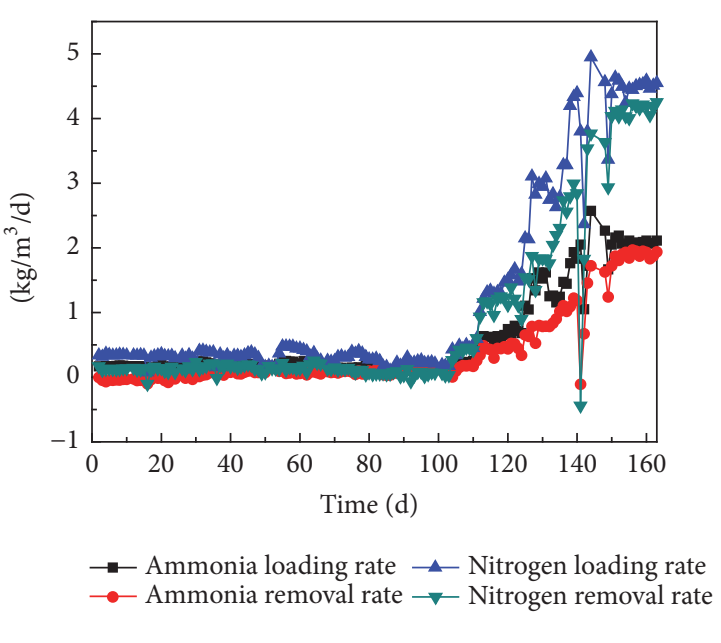

(b)

Figure 2: Continued. 

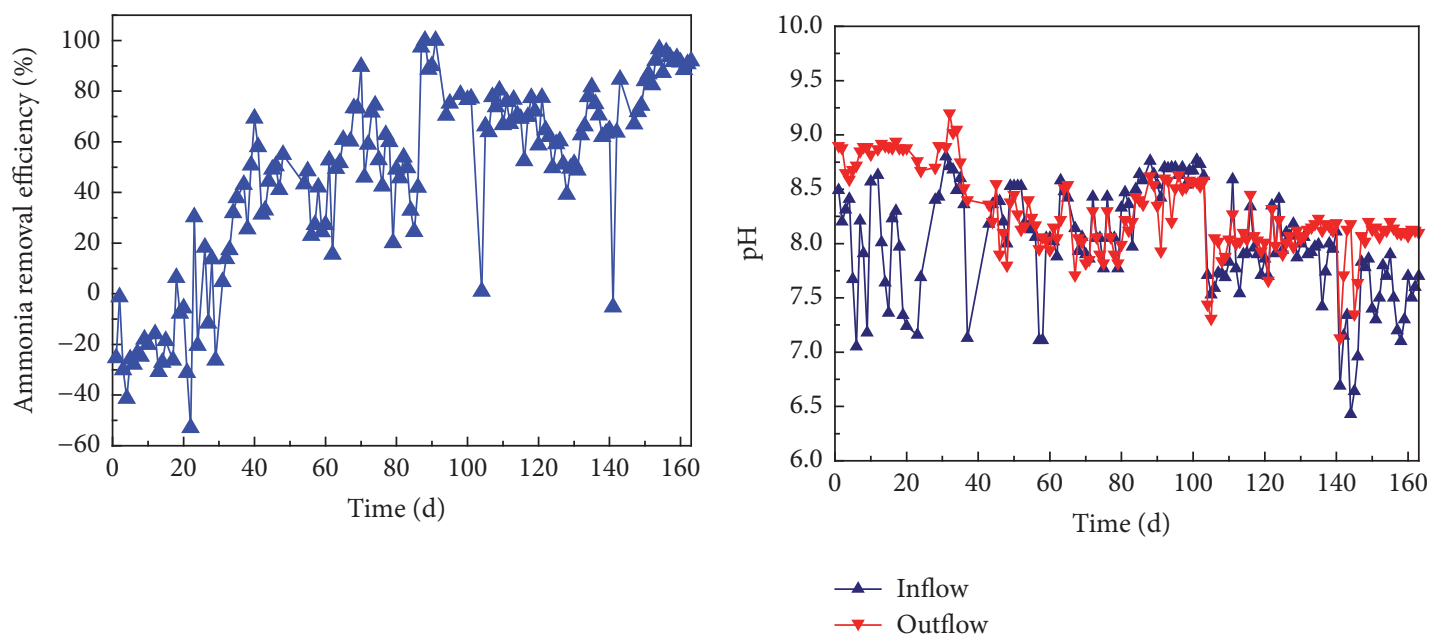

(c)

(d)

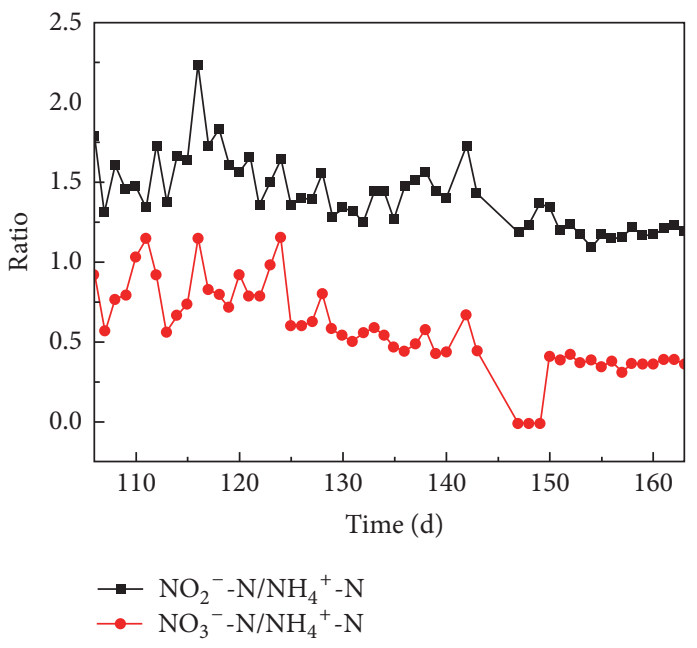

(e)

Figure 2: Performance of the anammox reactor.

\section{References}

[1] L. Xiong, Y.-Y. Wang, C.-J. Tang et al., "Start-up characteristics of a granule-based anammox UASB reactor seeded with anaerobic granular sludge," BioMed Research International, vol. 2013, Article ID 396487, 9 pages, 2013. 

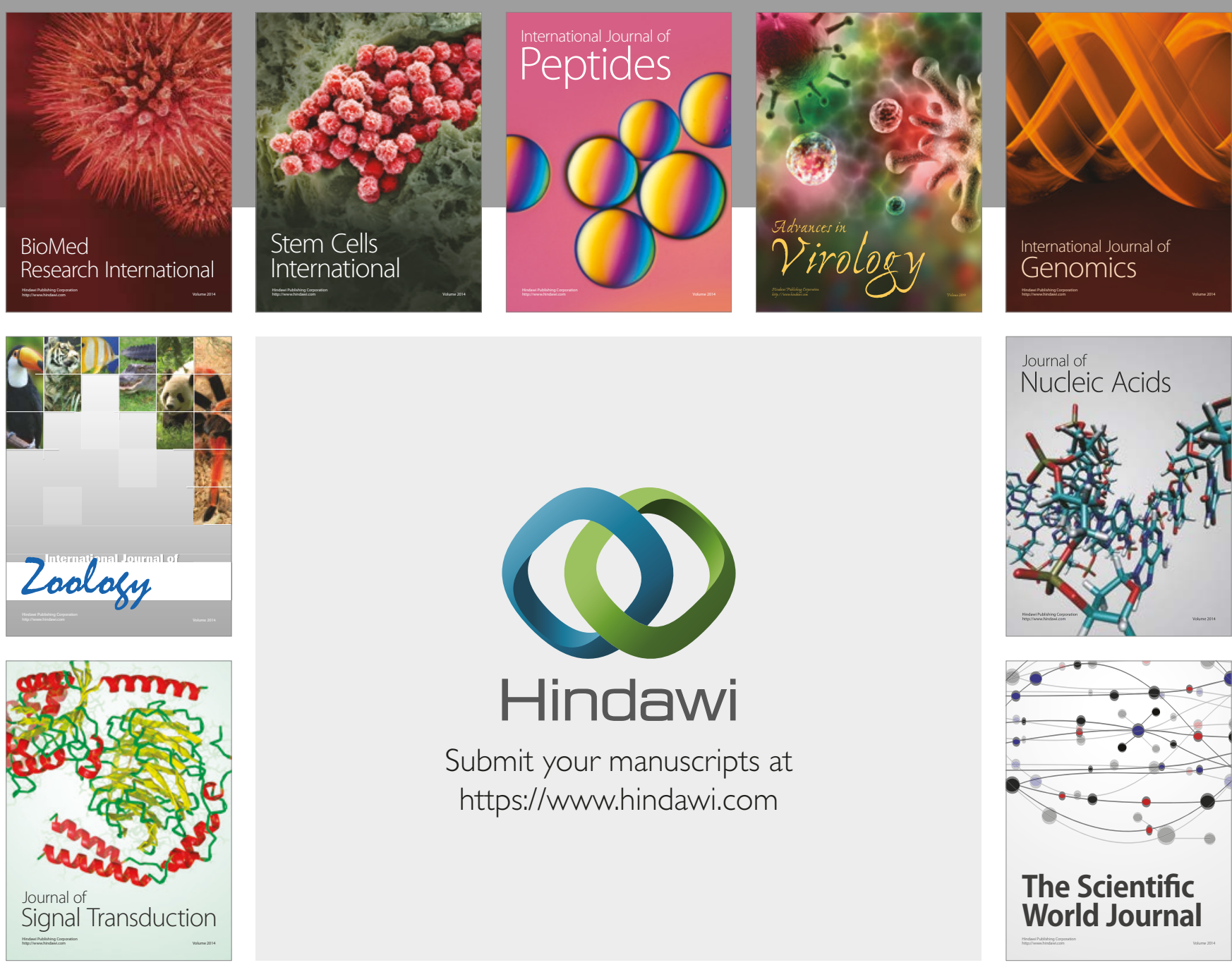

Submit your manuscripts at

https://www.hindawi.com
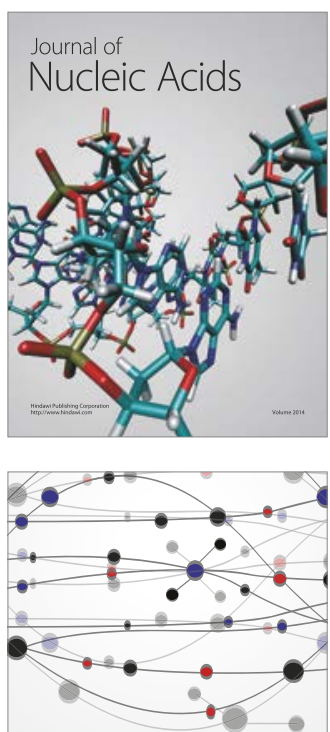

The Scientific World Journal

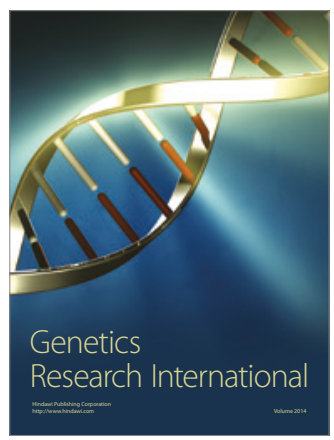

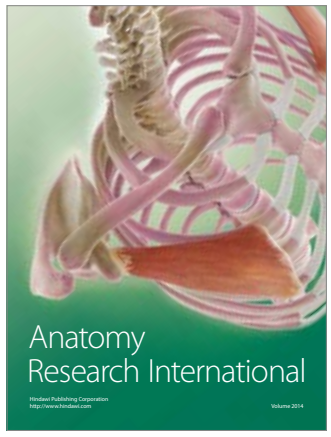

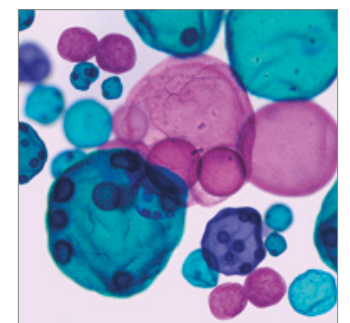

International Journal of Microbiology
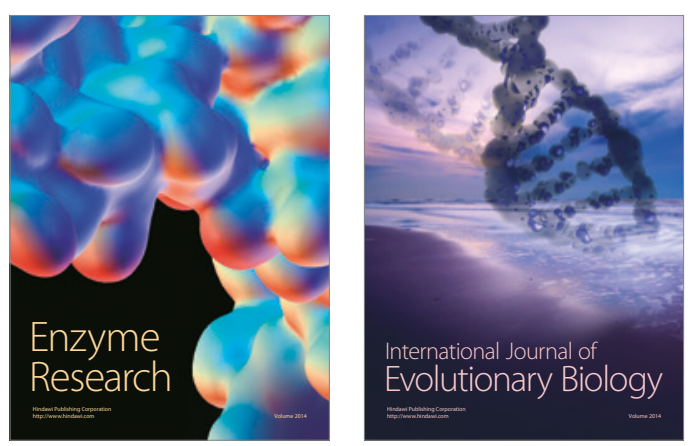
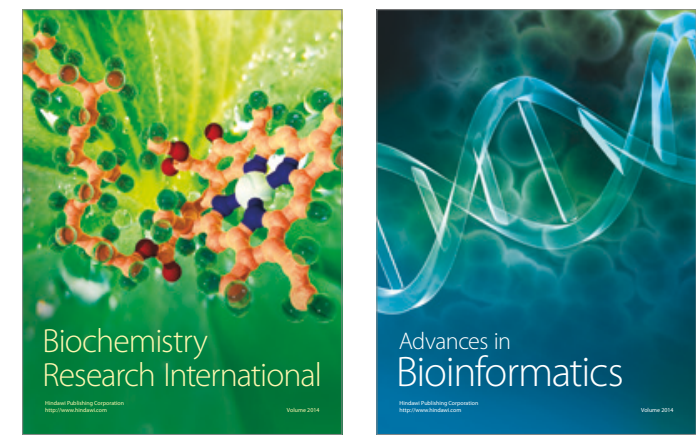

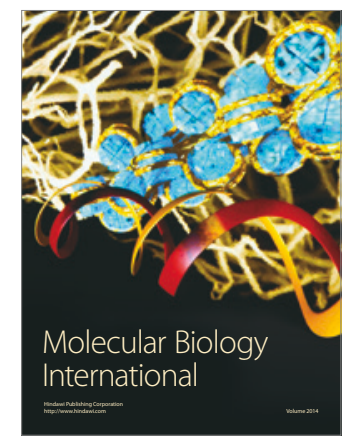

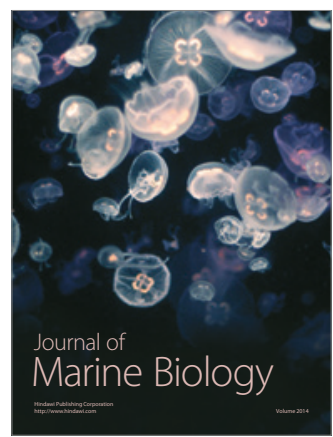

